\title{
COMPARING METHODS OF DETECTING MIND WANDERING WHILE DRIVING
}

\author{
Daniela Barragan, Daniel M. Roberts, Steven S. Chong, Carryl L. Baldwin \\ George Mason University \\ Fairfax, VA, USA \\ Email: dbarraga@gmu.edu
}

\begin{abstract}
Summary: Driver distraction is a persistent threat to traffic safety. External distraction has been examined extensively, but few studies have focused on internal distraction such as mind wandering. Equivocal results from the few existing studies are likely due, at least in part, to different experimental methods. Mind wandering is commonly assessed using either a self-caught or probe-caught method. The current investigation sought to better understand the effects of mind wandering on driving performance using the self-caught method and the probecaught method. In the Self-Caught Experiment, lateral control measures such as, lateral position variability and steering reversal rate were greater when drivers reported on-task thoughts versus mind wandering. In the Probe-Caught Experiment, these results were not replicated using the traditional probe-caught analysis. Instead, when analyzing the results of the Probe-Caught Experiment in a similar manner as the Self-Caught Experiment, the results were replicated. These results highlight methodological concerns in detecting mind wandering while driving. Additional research is needed to determine which method should be employed in future studies.
\end{abstract}

\section{OBJECTIVES}

On average, drivers are distracted $50 \%$ of the time while driving (Dingus et al., 2016). It is not uncommon for a driver to arrive at a destination, and then wonder how he or she made it there. This phenomenon, first termed highway hypnosis, was thought to occur when "the monotony of the surroundings and the necessity to attend only to a very small part of the visual field might induce some kind of hypnotic trance" (Williams, 1963 as cited in Wertheim, 1978, p. 111). This phenomenon is now commonly understood as mind wandering. Mind wandering is defined as "a shift of attention away from a primary task toward internal information" (Smallwood \& Schooler, 2006, p. 946).

Several recent studies have evaluated how mind wandering affects driving performance (Bencich, Gamboz, Coluccia, \& Brandimonte, 2014; He, Becic, Lee, \& McCarley, 2011; Yanko $\&$ Spalek, 2014). Of these studies, the primary mind wandering detection methods were either self-caught or probe-caught. Using the self-caught method, participants are instructed to press a response button as soon as they notice their mind wandering (He et al., 2011). Alternatively, using the probe-caught method, participants are instructed to press a response button to indicate whether their mind was wandering when they hear a probe tone (Bencich et al., 2014; Yanko \& Spalek, 2014). If, at this time, the participant's mind was not wandering, they are instructed to press an alternative button to indicate a state of alertness (or task-related thoughts).

He et al. (2011) used the self-caught method and found that variability in velocity increased when drivers were alert compared to mind wandering. They also found that lateral control (i.e., 
steering, lane deviation, lateral position variability) was unaffected by the driver's state of internal attention. Similarly, when using the probe-caught method, Bencich et al. (2014) found that speed and speed variability increased when drivers were alert compared to mind wandering. Conversely, Yanko and Spalek (2014), who also used the probe-caught method, found that participants drove at greater speeds when mind wandering compared to alert.

Given these inconsistent results, the purpose of the present investigation was to examine performance differences when drivers were actively thinking about the driving task (here referred to as an alert state) versus experiencing task-unrelated thoughts (here referred to as mind wandering) using two common detection methods, self-caught and probe-caught. Although researchers (e.g., Yanko \& Spalek, 2014) suggest that both detection methods are equally reliable, to our knowledge this has not been previously explored, at least in regard to driving.

One issue with the use of the self-caught method is that drivers' must be aware of their mind wandering in order to report it. Numerous studies, however, demonstrate that people are often not aware, at least for much of the time, that they are mind wandering (for a review see Schooler et al., 2011). Therefore, using a self-caught method may result in reduced reports of mind wandering relative to the probe-caught method. Further, those self-caught instances of mind wandering (when people have the meta-awareness of their mind wandering) may differ systematically from probe-caught instances. Specifically, providing probes are likely to bring drivers' awareness back to the task at hand, interferring with the overall nature of the task.

Based on the observation that focusing attention on automatized tasks frequently decreases performance (referred to as choking; see Ehrlenspiel, Wei, \& Sternad, 2010; Koedijker \& Mann, 2015), it was hypothesized, as assessed by operational control metrics, that driving performance would be less variable while mind wandering than alert. Specifically, it was expected that speed variability, lane deviation, lateral position variability and steering reversal rate would be lower when mind wandering. This finding would support the results of previous studies evaluating mind wandering while driving (Bencich et al., 2014; He et al., 2011). It was also hypothesized that performance decrements elicited by mind wandering would not differ using the two detection methods, but the frequency of reported mind wandering would be reduced in the SelfCaught Experiment.

\section{SELF-CAUGHT EXPERIMENT}

\section{Method}

Participants. Twenty students ( 9 men, 11 women) from George Mason University participated in the study for research credit. All participants were at least 18 years of age, held a valid driver's license, and had normal or corrected-to-normal vision and hearing. Visual acuity of at least 20/20 was verified using the Rosenbaum near vision test (Rosenbaum, 1984). On average, participants were $19.95(S D=4.43)$ years of age and had $4.00(S D=4.09)$ years of driving experience.

Apparatus. Participants completed a series of questionnaires assessing mind wandering and inattention, a demographics and driving history questionnaire, and the Karolinska Sleepiness 
Scale (KSS; Åkerstedt \& Gillberg, 1990). The KSS was administered twice, once prior to the experiment (along with the other questionnaires), and once after the experiment.

A medium fidelity driving simulator with a $240^{\circ}$ rotation steering wheel, and pedals was used in this study. The independent variables evaluated were attentional state (alert, mind wandering) and drive (drive one, drive two). As is common in previous studies (He et al., 2011), periods of mind wandering and alertness were evaluated via pairs of 10 -second windows pre and post selfcaught button press. Here, mind wandering was defined as the period of -13 to -3 seconds prior to the onset of the button press, while alertness was defined as the period of 3 to 13 seconds following the button press (see Figure 1).

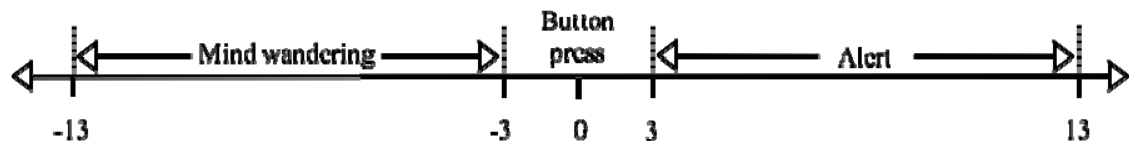

Figure 1. Self-caught mind wandering detection times

The dependent variables were average speed $(\mathrm{km} / \mathrm{h})$, speed variability (standard deviation of speed), lane deviation (root-mean-squared-error in meters from lane center), lateral position variability (standard deviation in meters of each participant's mean lateral position), and steering reversal rate (see He et al., 2011).

Procedure. Participants signed an informed consent form and completed a series of surveys. Participants then completed a practice drive ( 7 minutes), drive one (15 minutes), the sustained attention to response task (SART; Robertson, Manly, Andrade, Baddeley, \& Yiend, 1997) for 15 minutes, drive two (15 minutes), and then the second KSS. During the practice drive, participants were instructed to maintain a speed of $96.56 \mathrm{~km} / \mathrm{h}(60 \mathrm{mph})$, stay in the right lane, and when prompted, to exit the highway and proceed back onto the highway in the opposite direction. Following the practice drive, participants were instructed to read a mind wandering definition adapted from Singer and Antrobus (1972). The instructions for the two experimental drives were similar to the practice drive with one exception. Participants were instructed to press a button on the steering wheel when they noticed they were mind wandering (He et al., 2011; Smallwood \& Schooler, 2015). Between the experimental drives, participants completed the SART to induce cognitive fatigue (Robertson et al., 1997). Performance data on the SART is not presented here.

\section{Results}

Participants, on average, reported mind wandering $8.35(S E=.76)$ times in drive one, and 8.50 $(S E=.91)$ times in drive two, $t(19)=-0.20, p=.84$. The number of mind wandering instances reported for the curvy segments and straightaways were standardized based on the average time that participants drove in each segment. Participants reported significantly more instances of mind wandering during the curves compared to the straightaways, $t(18)=3.23, p=.005$. The results from linear mixed models are displayed in Table 1. 
Table 1. Linear mixed models using the self-caught method

\begin{tabular}{|c|c|c|c|c|c|c|}
\hline & \multicolumn{3}{|c|}{ State (mind wandering relative to alert) } & \multicolumn{3}{|c|}{ Drive (two relative to one) } \\
\hline & $t$ & $d f$ & $p$ & $t$ & $d f$ & $p$ \\
\hline Speed & .57 & 450.30 & .57 & 9.35 & 452.60 & $<.001$ \\
\hline Speed variability & -0.65 & 449.90 & .52 & 5.03 & 452.90 & $<.001$ \\
\hline Lane deviation & .46 & 450.70 & .65 & 4.48 & 457 & $<.001$ \\
\hline SDLP & -2.57 & 450.90 & .01 & 2.74 & 458.60 & .006 \\
\hline SRR* & -3.14 & - & .002 & 2.76 & - & .006 \\
\hline
\end{tabular}

*A Poisson mixed model was used to analyze SRR (reported as $z$ not $t$ )

The results from the Self-Caught Experiment are in line with He et al. (2011), such that speed variability was greater when drivers were alert $(M=.68, S E=.08)$ compared to mind wandering $(M=.61, S E=.07)$ using the self-caught method, though $p>.05$. SDLP and SRR were significantly greater when drivers were alert $\left(M_{s d l p}=.20, S E_{s d l p}=.01 ; M_{s r r}=.023, S E_{s r r}=.002\right)$ compared to mind wandering $\left(M_{s d l p}=.18, S E_{s d l p}=.01 ; M_{s r r}=.018, S E_{s r r}=.002\right), p<.05$. However, there was no significant effect of attentional state for speed, speed variability, and lane deviation. Speed, speed variability, lane deviation, SDLP, and SRR were significantly greater during drive two than drive one, $p<.05$.

\section{PROBE-CAUGHT EXPERIMENT}

\section{Method}

Participants. Twenty students (9 men, 11 women) from George Mason University participated in the study for research credit. All participants were at least 18 years of age, held a valid driver's license, and had normal or corrected-to-normal vision and hearing. Visual acuity of at least 20/20 was verified using the Rosenbaum near vision test (Rosenbaum, 1984). On average, participants were $21.55(S D=4.03)$ years of age and had $5.05(S D=3.52)$ years of driving experience.

Apparatus. This Probe-Caught Experiment was identical to the Self-Caught Experiment with the exception that periods of mind wandering were identified via the probe-caught, rather than selfcaught detection method. The onset of probe tones was jittered with a uniform distribution between 35 and 45 seconds, resulting in an average probe frequency of one per 40 seconds.

The independent and dependent variables remained the same; however, the attentional state (alert, mind wandering) variable was analyzed differently. Following previous probe-caught studies, mind wandering was evaluated as 10 seconds prior to the onset of a button press when the button press was used to indicate mind wandering, and alert was evaluated as 10 seconds prior to the onset of a button press when the button press was used to indicate alert (Yanko \& Spalek, 2014). Figure 2 provides an illustration of this analysis technique. 


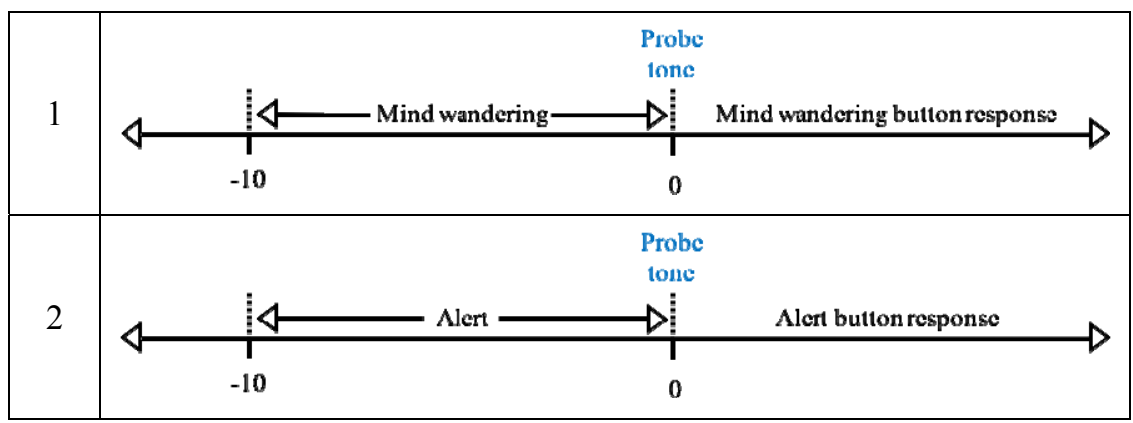

Figure 2. Probe-caught 1) mind wandering detection, and 2) alert detection

This detection technique assumes that participants reliably distinguish their current attentional state as either mind wandering or alert. Since the present study was interested in comparing the results for the Self-Caught and Probe-Caught Experiments, a novel analysis technique was also performed. For this detection technique, mind wandering was evaluated as 10 seconds prior to the onset of the probe tone, and for comparison, alert was evaluated as 13 seconds from the offset of a button press with a three second buffer.

Procedure. The only procedural difference from the Self-Caught Experiment was the mind wandering detection instructions. Once a probe tone was played, participants were instructed to indicate by pressing the corresponding button on the steering wheel whether they were mind wandering or alert when they heard the probe tone.

\section{Results}

Participants reported mind wandering $11.80(S E=1.29)$ times in drive one, and $13.95(S E=$ 1.34) times in drive two, $t(19)=-1.94, p=.07$. Of note, we could not directly compare the differences in the number of mind wandering instances during the curves and straightaways as was done in the Self-Caught Experiment because the probes were randomly presented and any differences would depend on the number of probes presented during the curves and straightaways. The results of linear mixed models using the traditional probe-caught analysis technique are displayed in Table 2.

Table 2. Linear mixed models using the traditional probe-caught analysis

\begin{tabular}{|c|c|c|c|c|c|c|}
\hline & \multicolumn{3}{|c|}{ State (mind wandering relative to alert) } & \multicolumn{3}{|c|}{ Drive (two relative to one) } \\
\hline & $t$ & $d f$ & $p$ & $t$ & $d f$ & $p$ \\
\hline Speed & .71 & 554.20 & .48 & 8.42 & 549.10 & $<.001$ \\
\hline Speed variability & -0.09 & 567.90 & .93 & 2.48 & 549.70 & .01 \\
\hline Lane deviation & 2.22 & 566.40 & .03 & 4.12 & 550.20 & $<.001$ \\
\hline SDLP & .24 & 567.20 & .81 & 2.33 & 549.40 & .02 \\
\hline SRR* & -0.66 & - & .51 & 4.02 & - & $<.001$ \\
\hline
\end{tabular}

*A Poisson mixed model was used to analyze SRR (reported as $z$ not $t$ )

Lane deviation was significantly greater when drivers were mind wandering $(M=.44, S E=.02)$ compared to alert $(M=.39, S E=.02), p=.03$. However, there was no significant effect of state 
for speed, speed variability, SDLP, and SRR. Speed, speed variability, lane deviation, SDLP, and SRR were significantly greater during drive two than drive one, $p<.05$. The results of linear mixed models evaluating pre- and post-probe data for mind wandering responses are displayed in Table 3.

Table 3. Linear mixed models using pre- and post-probe mind wandering responses

\begin{tabular}{|c|c|c|c|c|c|c|}
\hline & \multicolumn{3}{|c|}{ State (mind wandering relative to alert) } & \multicolumn{3}{|c|}{ Drive (two relative to one) } \\
\hline & $t$ & $d f$ & $p$ & $t$ & $d f$ & $p$ \\
\hline Speed & .84 & 704.10 & .40 & 8.79 & 705.20 & $<.001$ \\
\hline Speed variability & .27 & 703.80 & .79 & 2.73 & 707.30 & .006 \\
\hline Lane deviation & -0.71 & 705.20 & .48 & 3.41 & 709.90 & $<.001$ \\
\hline SDLP & -2.96 & 704.30 & .003 & 3.58 & 707.60 & $<.001$ \\
\hline SRR* & -3.07 & - & .002 & 4.35 & - & $<.001$ \\
\hline
\end{tabular}

*A Poisson mixed model was used to analyze SRR (reported as $z$ not $t$ )

The results for SDLP and SRR replicate that of the Self-Caught Experiment such that, SDLP and SRR were significantly greater when drivers were alert $\left(M_{s d l p}=.25, S E_{s d l p}=.02 ; M_{s r r}=.027\right.$, $\left.S E_{s r r}=.003\right)$ compared to mind wandering $\left(M_{s d l p}=.22, S E_{s d l p}=.02 ; M_{s r r}=.022, S E_{s r r}=.002\right), p$ $<.05$. However, there was no significant effect of state for speed, speed variability, and lane deviation. Speed, speed variability, lane deviation, SDLP, and SRR were significantly greater during drive two than drive one, $p<.05$.

Yanko and Spalek (2014) found that speed was greater when drivers were mind wandering compared to alert using the probe-caught method. However, when analyzing attentional state using the pre-probe data (same procedure as Yanko \& Spalek, 2014), our results did not support this. Interestingly, when analyzing the probe-caught data similar to the self-caught method, our results were in line with Yanko and Spalek (2014) such that, speed and speed variability trended towards being greater when drivers were mind wandering relative to when alert. However, these results in the current study were not significant.

\section{CONCLUSION}

The goal for this research was to better understand how driving performance is affected by mind wandering using two detection methods, self- and probe-caught. In the Self-Caught Experiment, lateral control measures including SDLP and SRR were significantly greater when drivers were alert relative to mind wandering. In the Probe-Caught Experiment, using the traditional analysis technique, these results were not found. Instead, using our novel analysis technique in the ProbeCaught Experiment, we replicated the results of the Self-Caught Experiment. These differing results, which were also identified in previous studies (Bencich et al., 2014; He et al., 2011; Yanko \& Spalek, 2014), raise concern about the validity of each method.

In the Self-Caught Experiment, participants reported more instances of mind wandering during curvy segments of the drive compared to the straightaways. It is possible that the curves served as an alerting function causing drivers to gain meta-awareness of their current attentional state. This potential bias in mind wandering reporting could contribute to the lower number of mind wandering instances reported in the Self-Caught Experiment relative to the Probe-Caught Experiment. Alternatively, in the Probe-Caught Experiment, it is possible that when prompted, 
participants had difficulty accurately reporting their current attentional state. These differing results could also be attributed to the different number of reported alert and mind wandering instances between detection methods. In the self-caught method, each participant has an equal number of mind wandering and alert instances. However, using the probe-caught method resulted in increased reports of mind wandering relative to alert states, and therefore the number of instances of mind wandering and alert are unbalanced. The present study attempted to account for this unbalanced data and potential individual differences by treating the participant variable as a random effect using linear mixed models (Kliegl, Wei, Dambacher, Yan, \& Zhou, 2011). The present study highlights methodological issues in detecting mind wandering while driving and warrants future research to determine which method most accurately depicts the driver's attentional state, whether alert or mind wandering.

\section{REFERENCES}

Åkerstedt, J., \& Gillberg, M. (1990). Subjective and objective sleepiness in the active individual. The International Journal of Neuroscience, 52(1-2), 29-37.

Bencich, E., Gamboz, N., Coluccia, E., \& Brandimonte, M. A. (2014). 'When the mind flies': the effects of mind-wandering on driving. Proceedings of the Trieste Symposium on Perception and Cognition, San Giovanni in Fiore, Italy, 91-93.

Dingus, T. A., Guo, F., Lee, S., Antin, J. F., Perez, M., Buchanan-King, M., \& Hankey, J. (2016). Driver crash risk factors and prevalence evaluation using naturalisic driving data. Proceedings of the National Academy of Sciences, 113(10), 2636-2641.

Ehrlenspiel, F., Wei, K., \& Sternad, D. (2010). Open-loop, closed-loop and compensatory control: Performance improvement under pressure in a rhythmic task. Experimental Brain Research, 201(4), 729-741. doi: 10.1007/s00221-009-2087-8

He, J., Becic, E., Lee, Y.-C., \& McCarley, J. S. (2011). Mind wandering behind the wheel: Performance and oculomotor correlates. Human Factors, 53(1), 13-21.

Kliegl, R., Wei, P., Dambacher, M., Yan, M., \& Zhou, X. (2011). Experimental effects and individual differences in linear mixed models: Estimating the relationship between spatial, object, and attraction effects in visual attention. Frontiers in Psychology: Quantitative Psychology and Measurement, 1(238), 1-12.

Koedijker, J., \& Mann, D. (2015). Consciousness and choking in visually-guided actions. Phenomenology and the Cognitive Sciences, 14(2), 333-348. doi: 10.1007/s11097-014-93983

Robertson, I. H., Manly, T., Andrade, J., Baddeley, B. T., \& Yiend, J. (1997). 'Oops!': Performance correlates of everyday attentional failures in traumatic brain injured and normal subjects. Neuropsychologia, 36(6), 747-758.

Rosenbaum, J. G. (1984). The biggest reward for my invention isn't money. Journal of Medical Economy, 61, 152-163.

Schooler, J. W., Smallwood, J., Christoff, K., Handy, T. C., Reichle, E. D., \& Sayette, M. A. (2011). Meta-awareness, perceptual decoupling and the wandering mind. Trends in Cognitive Sciences, 15(7), 319-326. doi: 10.1016/j.tics.2011.05.006 
Singer, J. L., \& Antrobus, J. S. (1972). Daydreaming, imaginal processes, and personality: A normative study. The nature and function of imagery (P.W. Sheehan Ed.). New York: Academic Press, 175-202.

Smallwood, J., \& Schooler, J.W. (2006). The restless mind. Psychological Bulletin, 132(6), 946958.

Smallwood, J., \& Schooler, J.W. (2015). The science of mind wandering: Empirically navigating the stream of consciousness. Annual Review of Psychology, 66(6), 487-518.

Wertheim, A.H. (1978). Explaining highway hypnosis: Experimental evidence for the role of eye movements. Accident Analysis and Prevention, 10, 111-129. doi:10.1016/00014575(78)90019-1

Williams, G. W. (1963). Highway hypnosis: An hypothesis. International Journal of Clinical and Experimental Hypnosis, 11(3), 143-151.

Yanko, M. R., \& Spalek, T. M. (2014). Driving with the wandering mind: The effects that mindwandering has on driving performance. Human Factors, 56(2), 260-269. 